\title{
Enfoque en el diagnóstico diferencial de las Taquicardias Paroxísticas Supraventriculares en el Laboratorio de electrofisiología
}

\author{
Mauricio Moreno ${ }^{1}$,Rodrigo $\mathrm{Isa}^{2}$ \\ 1. Clínica Santa María, Clínica Dávila \\ 2. Clínica Tabancura \\ Sin conflictos de financiamiento.
}

Las taquicardias paroxísticas supraventriculares son arritmias frecuentes y producen importante morbilidad. El estudio electrofisiológico permite hacer el diagnóstico su mecanismo para luego realizar la ablación. El diagnóstico no siempre es sencillo y se debe recurrir a múltiples observaciones y maniobras para alcanzarlo. En la siguiente revisión se discuten los principales criterios usados para el diagnóstico del mecanismo de estas taquicardias durante un estudio electrofisiológico.

Palabras Clave: Taquicardia paroxística supraventricular, Arritmia.

\section{Differential diagnosis of Paroxysmal Supraventricular Tachycardia at the Electrophysiology laboratory}

Paroxysmal supraventricular tachycardias are frequently observed arrhythmias associated to significant morbidity. Electrophysiological study allows the diagnosis of the mechanisms underlying the arrhythmia leading toblation. The diagnosis is not always easy and multiple observations and maneuvers are required to uncover it. In the following review, the main criteria used to diagnose the mechanisms of these tachycardias during an electrophysiological study are discussed.

Keywords: Supraventricular paroxysmal tachycardias, Arrhythmias 


\section{Introducción:}

La taquicardia paroxística supraventricular (TPSV) es una arritmia frecuente, que a menudo produce consultas en los servicios de urgencia y genera una alta morbilidad a los pacientes. Para prevenir los episodios de taquicardia se puede indicar antiarrítmicos; sin embargo, estos tienen efectos adversos y es inapropiado un tratamiento diario para un evento de ocurrencia aleatoria. Por ello, la mayoría de los pacientes se tratan con ablación de la taquicardia, que es generalmente curativo, con una alta tasa de éxito y bajas complicaciones. Antes de la ablación se efectúa el estudio electrofisiológico (EEF) que determinará el mecanismo exacto de la taquicardia. Este diagnóstico puede ser sencillo en algunos casos, pero en otros necesitamos de observaciones y maniobras precisas para aclararlo.

Las TPSV tienen 3 mecanismos: taquicardia por reentrada intranodal (TIN), taquicardia aurículo-ventricular mediada por una vía accesoria (TAV) y taquicardia auricular (TA). Otra entidad es la taquicardia ectópica de la unión AV (JET), con mayor incidencia en la población pediátrica y comportamiento similar a la TA. El diagnóstico diferencial entre estas arritmias se inicia con el cuadro clínico, se puede sugerir con el electrocardiograma (ECG) y, finalmente, el diagnóstico definitivo se establece con el EEF.

En la siguiente revisión se analizarán los criterios usados durante un EEF para el diagnóstico de las TPSV. El enfoque se basa en el análisis del electrocardiograma (ECG) basal y en taquicardia, los criterios electrofisiológicos observados durante taquicardia y las maniobras de estimulación en taquicardia. Hay que recordar que no existen criterios categóricos en el diagnóstico del mecanismo de una taquicardia, por lo cual se requiere la ponderación de los criterios observados.

\section{Características basales}

Los elementos del ECG que pueden orientar al diagnóstico de una TPSV son:

1. Si se observa un síndrome de preexcitación en el ECG el diagnóstico más probable es TAV. Este hallazgo tiene una especificidad del 97\% para la TAV; sin embargo, sólo se presenta en el $15 \%$ de los pacientes ${ }^{1}$.

2. Si durante taquicardia puede observarse una seudoR en la derivación $\mathrm{V} 1$ y menos frecuente una seudo $\mathrm{Q}$ en las derivaciones de la pared inferior, que representan la actividad auricular retrograda fusionada con el QRS, ello es característico de la TIN ${ }^{2}$.

\section{Características de la taquicardia en el EEF}

En la TIN hay un circuito reentrante que involucra el tejido peri-nodo aurículo-ventricular (AV), con participación de una vía nodal lenta (brazo anterógrado del circuito) y una vía nodal rápida (brazo retrogrado del circuito), con independencia de este circuito tanto de las aurículas como de los ventrículos. Esta reentrada circunscrita explica la activación casi simultánea de aurículas y ventrículos, que determina habitualmente que la TIN típica tenga un intervalo ventrículo-auricular (VA) $<70 \mathrm{mseg}$. Si el brazo retrógrado del circuito es una vía lenta, se puede generar un intervalo VA más prolongado y esto constituirá una TIN atípica. La inducción de la TIN en general se logra con un extra estímulo que se conduce por la vía lenta, permite la recuperación de la refractariedad de la vía rápida y así se forma el circuito reentrante. La presencia de esta fisiología de doble vía nodal se reconoce con la estimulación auricular programada, por presencia de un salto nodal cuando al reducir en 10 mseg el extra estímulo hay un aumento del intervalo $\mathrm{AH}$ en $50 \mathrm{mseg}$.

La localización más frecuente de las vías accesorias (VACC) es en el anillo mitral lateral, aproximadamente, el 60\% de los casos, y en ellas la activación auricular retrograda en el seno coronario durante taquicardia ocurre de distal a proximal (activación excéntrica). Mientras que cuando la ubicación de la VACC es septal o lateral derecha la activación auricular retrograda en el seno coronario será de proximal a distal (concéntrica).

El diagnóstico de la TA habitualmente requiere un esfuerzo adicional respecto a la observación de la taquicardia y las maniobras diagnósticas que se describirán posteriormente.

A veces, con la sola observación de las características de la taquicardia en el EEF suele ser suficiente para hacer el diagnóstico de su mecanismo. Pero hay casos más complejos, como aquellas TPSV que cursan con RP > PR (RP largo) en el ECG; en estos casos, hay variaciones en los mecanismos de la taquicardia que explican esta actividad auricular retrograda mas tardía. Cuando hay RP largo las opciones son: TA, TIN atípica y TAV con conducción retrógrada decremental por la VACC. En estos casos las observaciones y maniobras del EEF son esenciales para el diagnóstico correcto.

Los criterios a evaluar en el EEF en condiciones basales 


\section{Tabla 1: EEF basal}

\begin{tabular}{|c|c|c|}
\hline Hallazgo & Interpretación & Problema \\
\hline Intervalo HV $<35$ mseg & Sugiere TAV & Descartar rama derecha, VACC puede ser un bystander \\
\hline Ausencia de conducción VA & Descarta TAV & Conducción de la VACC puede mejorar con catecolaminas \\
\hline Activación auricular retrograda excéntrica & Sugiere TAV & VACC puede ser un bystander \\
\hline Fisiología de doble vía noda I & Sugiere TIN & No descarta otro mecanismo \\
\hline Respuesta extranodal a pacing parahisiano & Sugiere TAV & Principal utilidad en VACC septales y derechas \\
\hline $\begin{array}{l}\text { VA del pacing base del ventrículo derecho } \\
\text { (VD) < VA pacing ápex del VD }\end{array}$ & Sugiere TAV & Principal utilidad en VACC posteroseptales \\
\hline
\end{tabular}

y durante taquicardia se resumen en las Tablas 1 y 2

\section{Maniobras durante taquicardia}

Cuando el diagnóstico aún no es claro con estas observaciones, deben efectuarse maniobras durante taquicardia para aclarar el diagnóstico (Tabla 3).

\section{Discusión:}

En general, no existe un único criterio absoluto para el diagnóstico de las TPSV y es necesario todos los criterios en conjunto. Por ejemplo, cuando hay fisiología de doble vía nodal el valor predictivo positivo para la TIN es aproximadamente $86 \%$, porque de estos pacientes un $6 \%$ tiene una TAV y un $8 \%$ tiene una $\mathrm{TA}^{1}$. La estimulación auricular programada es la maniobra habitual para evaluar la presencia de fisiología de doble vía nodal. La estimulación auricular gatillada por el ventrículo con decremento progresivo del intervalo VA también ha sido reportada como útil para el diagnóstico de doble vía nodal ${ }^{3}$. Por otra parte, los pacientes con preexcitación pueden tener una TIN hasta en $10 \%$ de los $\operatorname{casos}^{1}$. La ausencia de conducción VA no descarta una VACC y puede hacerse evidente con la administración de catecolaminas. Hasta un $5 \%$ de casos con bloqueo VA con ciclos de estimulación ventricular $>600$ mseg tienen una $\mathrm{TAV}^{4}$. Hay maniobras sencillas que permiten evidenciar una VACC septal, como un intervalo VA más corto con pacing basal que con pacing desde el ápex del VD, aunque esta maniobra tiene limitaciones para las vías accesorias anteroseptales y eventualmente en presencia de bloqueo de rama derecha ${ }^{5}$. Cuando realizamos pacing cercano al His con alta salida podemos capturar tanto el His como la zona muscular del VD y así podremos medir la conducción retrograda auricular (intervalo estímulo-A); al reducir la salida del pacing se pierde la captura del His y si no hay cambio en la secuencia auricular retrograda y tampoco en el intervalo estímulo-A (respuesta extranodal), nos sugerirá VACC septal o de pared libre de $\mathrm{VD}^{6}$.

La inducción de la taquicardia con un AH crítico es un criterio de menor valor. En la TIN el inicio de la taquicardia depende del bloqueo anterógrado de la vía rápida y un suficiente retardo en la vía lenta para permitir la conducción retrograda por la vía rápida. Pero algunas TA sólo se inducen con un ciclo de estimulación que determina un $\mathrm{AH}$ largo lo que puede sugerir que se necesita un AH crítico. Además, en la TAV es necesario un retardo en la conducción AV que permita la conducción

Tabla 2: EEF en taquicardia

\begin{tabular}{|l|c|c|}
\hline Hallazgo & Interpretación & Problema \\
\hline Inducción con AH crítico & Sugiere TIN & No descarta TA ni TAV \\
\hline Activación auricular retrograda excéntrica & Sugiere TAV & VACC puede ser un bystander, TIN con salida izquierda, TA izquierda \\
\hline Bloqueo AV $(\mathrm{A}>\mathrm{V})$ & Sugiere TA & No descarta TIN con bloqueo AV \\
\hline Terminó de la taquicardia con bloqueo AV & Descarta TA & No diferencia TIN vs TAV \\
\hline Bloqueo de rama produce un aumento del VA $>20$ mseg & Sugiere TAV & Se observa solo en VACC ipsilaterales al bloqueo de rama \\
\hline
\end{tabular}


Tabla 3: Maniobras durante taquicardia

\begin{tabular}{|c|c|c|}
\hline Maniobra & Observación & Interpretación \\
\hline \multirow{2}{*}{$\begin{array}{l}\text { Primer intervalo VA post } \\
\text { sobrestimulación auricular }\end{array}$} & Fijo (igual al VA de la taquicardia) & Sugiere TIN o TAV \\
\hline & Variable (diferente al VA de la taquicardia) & Sugiere TA \\
\hline \multirow[t]{2}{*}{ Extraestímulo ventricular con His refractario } & Termina la taquicardia sin adelantar la aurícula & Descarta una TA y sugiere una TAV \\
\hline & Adelanta o retrasa la aurícula & Descarta una TA y sugiere una TAV \\
\hline \multirow{2}{*}{$\begin{array}{l}\text { Sobreestimulación ventricular a } \\
200-250 \text { mseg ( } 3 \text { a } 6 \text { latidos) }\end{array}$} & Se disocia el ventrículo y no terminó la taquicardia & Descarta TAV \\
\hline & Termina la taquicardia sin adelantar la aurícula & Descarta TA \\
\hline \multirow[t]{3}{*}{ Sobreestimulación ventricular } & No se encarrila la aurícula & Menos probable una TAV \\
\hline & $\begin{array}{l}\text { Se encarrila la aurícula con una activación } \\
\text { retrógada distinta }\end{array}$ & Menos probable una TAV \\
\hline & Se encarrila la aurícula y hay una respuesta V-A-A-V & Sugiere una TA \\
\hline \multirow[t]{3}{*}{ Encarrilar la taquicardia desde el ventrículo } & Ciclo de retorno $<115 \mathrm{mseg}$ & Sugiere TAV \\
\hline & Ciclo de retorno corregido $<110 \mathrm{mseg}$ & Mayor utilidad en VACC septales \\
\hline & $\begin{array}{l}\text { Ciclo de retorno > } 115 \text { mseg e Intervalo SA - VA > } \\
85 \text { mseg }\end{array}$ & Útil en TIN atípica \\
\hline
\end{tabular}

retrograda por la VACC, también sugiriendo la necesidad de un AH crítico.

Al contrario, el intervalo VA durante taquicardia es de gran valor diagnóstico: cuando es $<70 \mathrm{mseg}$ sugiere fuertemente una TIN y es capaz de descartar una TAV incluso aquellas con localización septal ${ }^{7}$. También, un VA $<70$ mseg permite descartar una TA aunque teóricamente es factible un VA corto en su presencia.

En el EEF hay hallazgos útiles pero infrecuentes de observar, como el bloqueo de rama con prolongación del intervalo $\mathrm{VA}^{8}$ o el bloqueo AV durante taquicardia, los cuales sugieren TAV y TA, respectivamente. Sin embargo, el bloqueo AV durante taquicardia también puede observarse en la TIN debido al bloqueo del tronco común inferior. El término de la taquicardia con A (bloqueo AV) descarta una TA, pero solo se observa en el $28 \%$ de las TPSV 1.

Las maniobras de estimulación auricular durante taquicardia son menos valiosas en el diagnóstico de las TPSV. Un intervalo VA variable post estimulación auricular durante taquicardia apoya fuertemente una TA, pero también se puede observar en las TIN $^{9}$. Ocasionalmente, en las TA se puede observar por coincidencia un intervalo VA fijo. Además, estas maniobras no permiten diferenciar entre TIN y TAV.

En cambio, las maniobras de estimulación ventricular durante taquicardia son de gran apoyo para el diagnóstico de las TPSV. La introducción de un extra estímulo ventricular en taquicardia con His refractario y que afecta la aurícula tiene una alta especificidad para una $\mathrm{TAV}^{1}$. El encarrilamiento ventricular de la taquicardia con una respuesta $\mathrm{V}-\mathrm{A}-\mathrm{V}$ prácticamente excluye una TA y es una maniobra que se puede aplicar en el $78 \%$ de las TPSV $^{1}$. La respuesta V-A-A-V es propia de la TA (Figura 1); sin embargo, debe excluirse una respuesta seudo $\mathrm{V}-\mathrm{A}-\mathrm{A}-\mathrm{V}$ donde el diagnóstico puede ser una TIN (Figura 2). El ciclo de retorno medido en el sito de pacing del ápex del ventrículo derecho es una maniobra sencilla que nos permite diferenciar una TIN de una TAV, y, si se hace la corrección de la conducción anterógrada del nodo AV), será más acucioso para el diagnóstico de las VACC septales (Figura 3) ${ }^{10}$. Tam- 
Figura 1. Estimulación ventricular a $390 \mathrm{mseg}$ con encarrilamiento de la taquicardia, se adelantan las aurículas a $390 \mathrm{mseg}$ y el ciclo de la taquicardia es 405 mseg. Se observa respuesta V-A-A-V compatible con una TA.

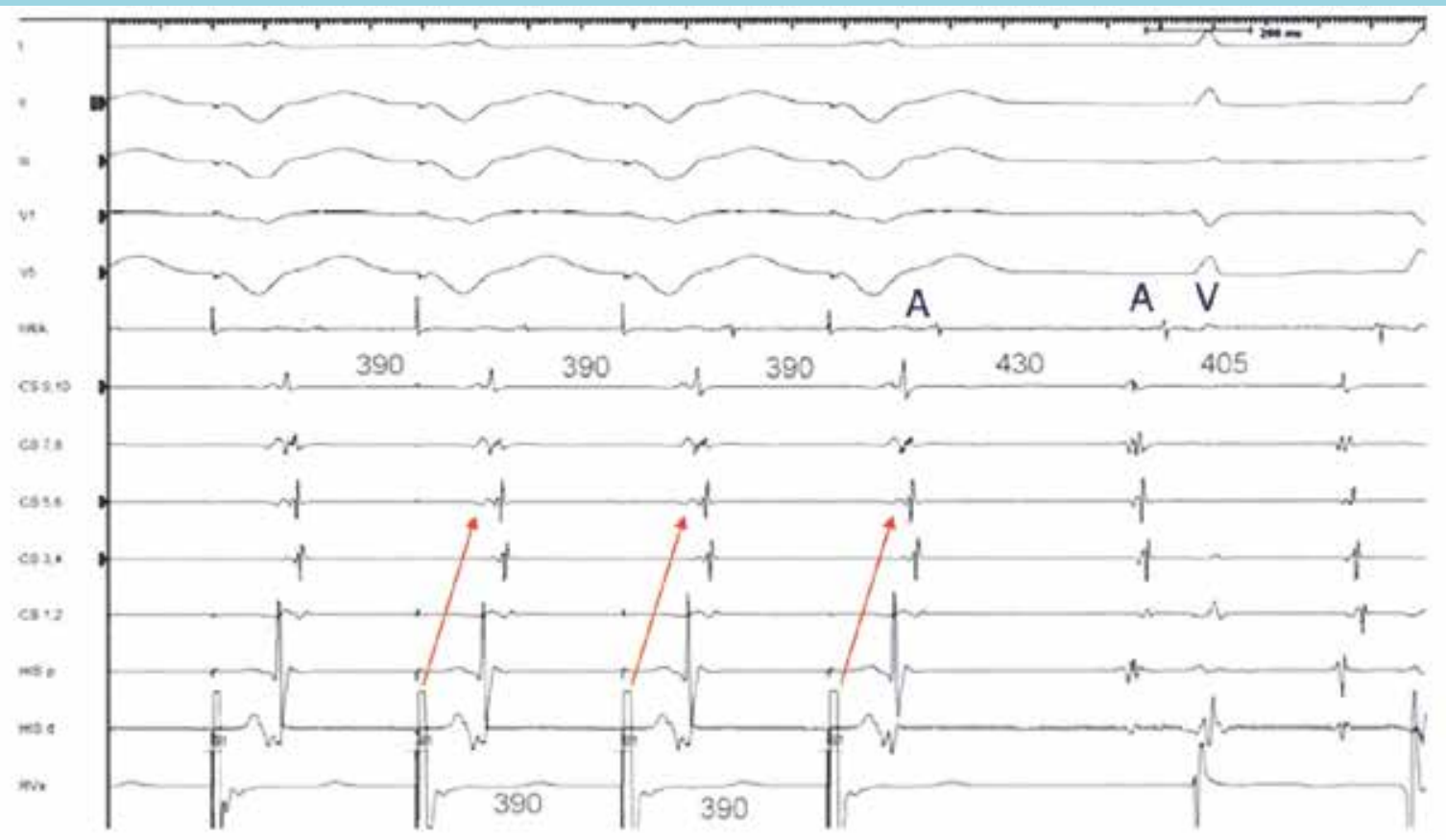

Figura 2. Estimulación ventricular a $230 \mathrm{mseg}$ con encarrilamiento de la taquicardia (ciclo de $250 \mathrm{mseg}$ ). Se observa una seudorespuesta A- A-V por actividad auricular retrograda tardía (líneas negras). Con intervalo post pacing de 460 mseg (línea azul) y ciclo de retorno de 210 mseg (460 - $250 \mathrm{mseg}$ ) compatible con TIN.

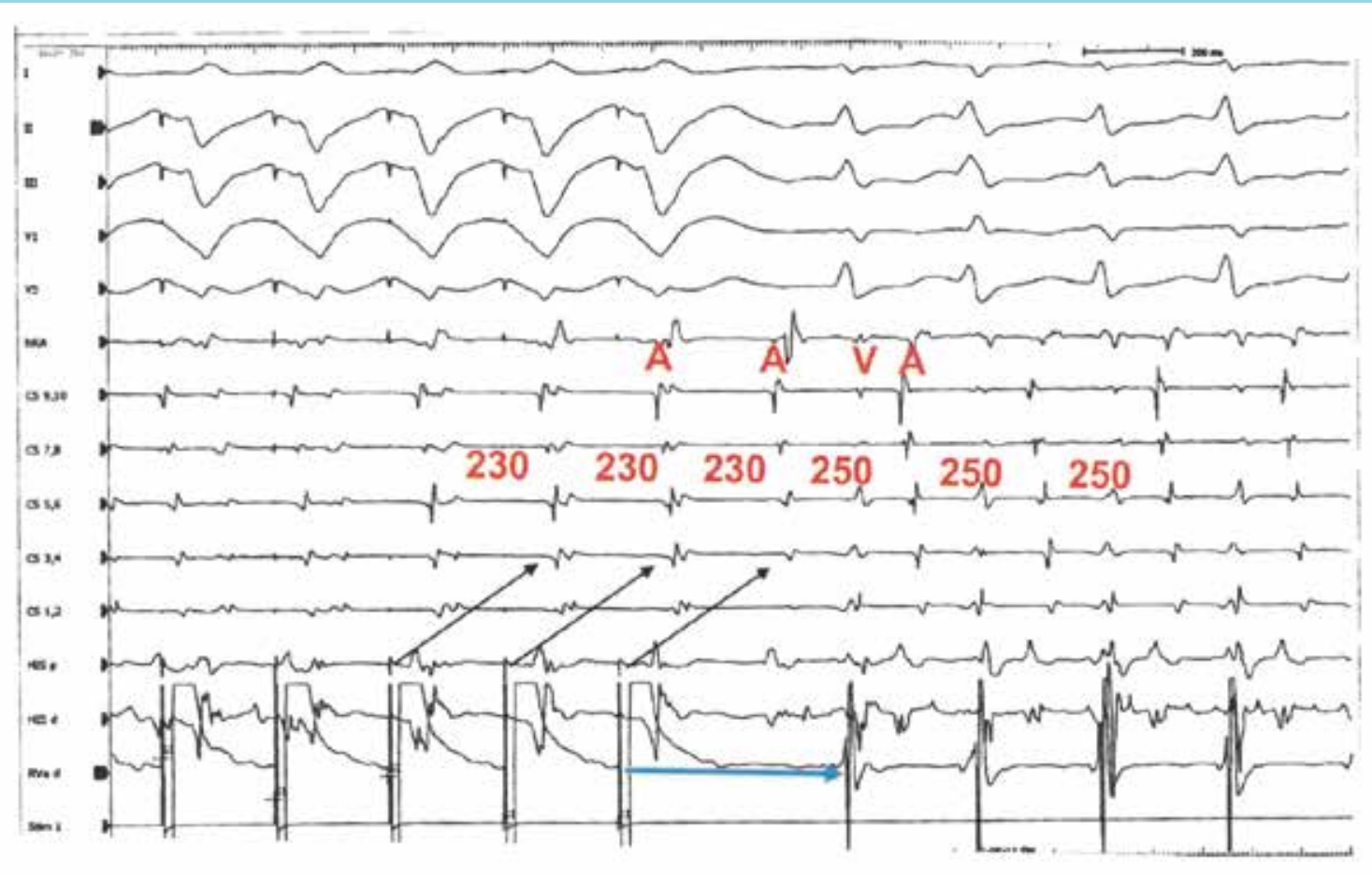



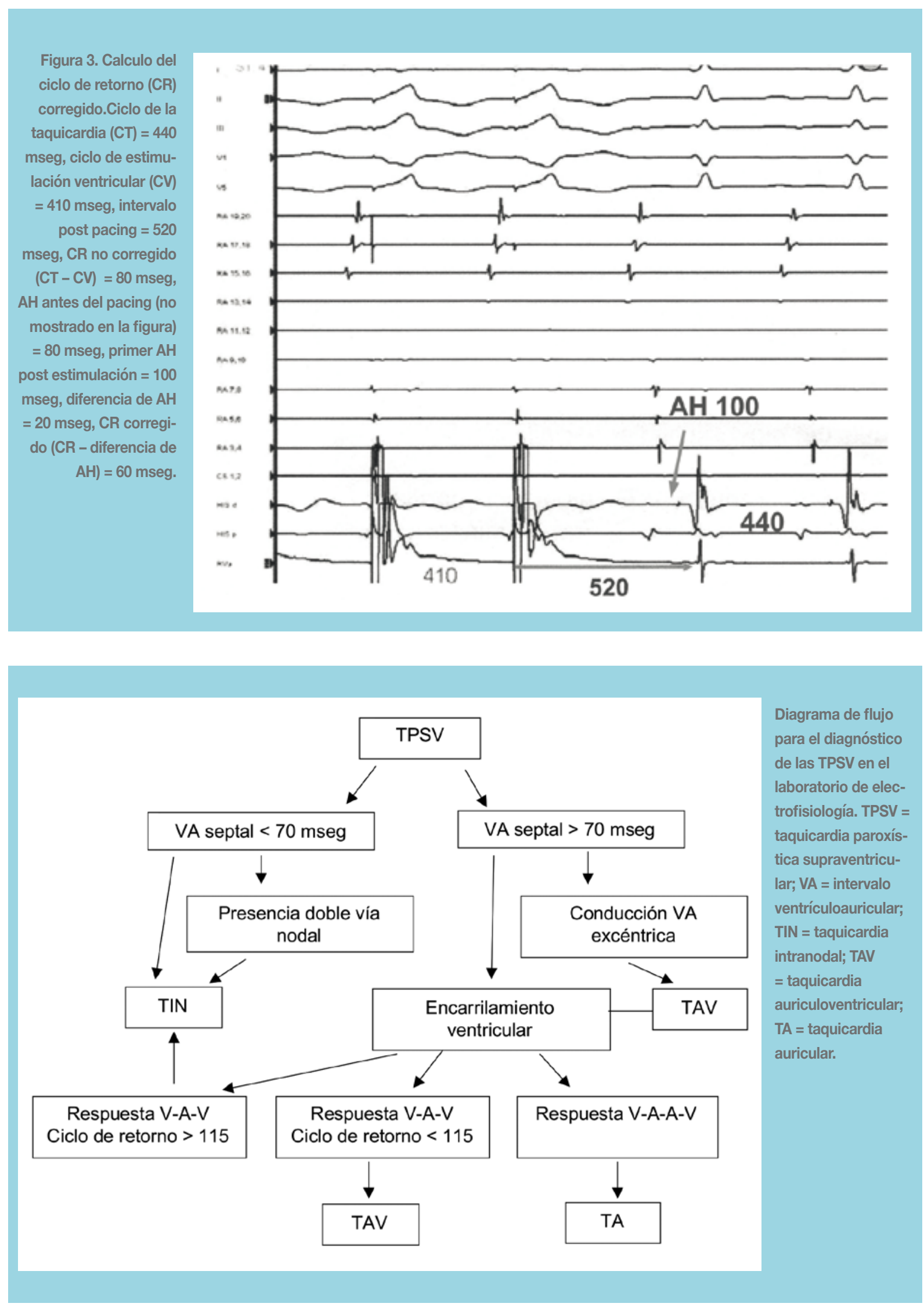
bién se puede medir el intervalo entre el último latido ventricular estimulado a la última aurícula encarrilada (SA), menos el intervalo VA de la taquicardia: cuando es $>85$ mseg sugiere una TIN, y junto a un ciclo de retorno $>115 \mathrm{mseg}$, son muy útiles en el diagnóstico de TIN atípica ${ }^{11}$. Cuando con estimulación ventricular rápida terminamos la taquicardia sin afectar la aurícu- la, prácticamente excluimos una TA, pero esto sólo se observa en el $27 \%$ de los casos 1 .

Finalmente, el esquema ilustrado representa un diagrama de flujo que puede ayudar en forma sencilla al diagnóstico diferencial de las TPSV en el laboratorio de electrofisiología.

\section{Referencias}

1. KNIGHT BP, EBINGER M, ORAL H, KIM MH, STICHERLING C, PELOSI F, MICHAUD GF, STRICKBERGER SA, MORADY F. Diagnostic value of tachycardia features and pacing maneuvers during paroxysmal supraventricular tachycardia. J Am Coll Cardiol 2000; 36: 574 - 82.

2. MORENO M, KARMELIC C, PUCCIO JM. Utilidad de las precordiales derechas para el diagnostico diferencial de las taquicardias paroxísticas. Revista Chilena de Cardiología 2005; 24: 438 .

3. DECHATEAU J, TIXIER R, VLACHOS K, et al. Ventricular-triggered atrial pacing: A new maneuver for slow-fast atrioventricular nodal reentrant tachycardia. Heart Rhythm 2020; 17: $955-964$

4. YAMAMOTO T, YEH SJ, LIN FC, WU DL. Effects of isoproterenol on accessory pathway conduction in intermittent or concealed Wolff-Parkinson-White syndrome. Am J Cardiol 1990; 65: $1438-42$.

5. MARTÍNEZ-ALDAY J, ALMENDRAL J, ARENAL A, ORMAETXE. JM, PASTOR A, VILLACASTÍN JP, MEDINA O, PEINADO R, DELCÁN J. Identification of concealed posteroseptal Kent pathways by comparation of ventriculoatrial intervals from apical and posteroseptal right ventricular sites. Circulation 1994; 89: 1060 - 1067.
6. HIRAO K, OTOMO K, WANG X, et al. Para-Hisian pacing: a new method for differentiating retrograde conduction over an accessory $\mathrm{AV}$ pathway from conduction overt the $\mathrm{AV}$ node. Circulation 1996; 94: 1027 - 35.

7. ROSS DL, UTHER JB. Diagnosis of concealed accessory pathway in supraventricular tachycardia. Pacing Clin Electrophysiol 1984; 7: $1069-85$.

8. KERR CR, GALLAGHER JJ, GERMAN LD. Changes in ventriculoatrial intervals with bundle branch block aberration during reciprocating tachycardia in patients witch accessory atrioventricular pathways. Circulation 1982; 66: $196-201$.

9. KNIGHT BP, ZIVIN A, SOUZA J, et al. A technique for the rapid diagnosis of atrial tachycardia in the electrophysiology laboratory. J Am Coll Cardiol 1999; 33: 775 - 81.

10. GONZALEZ-TORRECILLA E, ARENAL A, ATIENZA F, OSCA J, GARCÍA-FERNÁNDEZ J, PUCHOL A, SANCHEZ A, ALMENDRAL J. FIRST postpacing interval after tachycardia entrainment with correction for atrioventricular node delay: A simple maneuver for diferential diagnosis of atrioventricular nodal reentrant tachycardia versus orthodromic reciprocating tachycardias. Heart Rhythm 2006; 3: 674 - 679.

11. ABEDIN Z, CONNER R. Essential cardiac electrophysiology with self assessment 2007. 